\title{
Severe Human Bocavirus- Associated Pneumonia in Adults at a Referral Hospital, Seoul, South Korea
}

\author{
Sang-Ho Choi, ${ }^{1}$ Jin Won Huh, ${ }^{1}$ Sang-Bum Hong, Jiwon Jung, Min Jae Kim, \\ Yong Pil Chong, Sung-Han Kim, Heungsup Sung, Eun Jin Chae, Kyung-Hyun Do, \\ Sang-Oh Lee, Chae-Man Lim, Yang Soo Kim, Jun Hee Woo, Younsuck Koh
}

We report a case series of severe human bocavirus-associated pneumonia in adults in Seoul, South Korea. The virus accounted for $0.5 \%$ of all severe pneumonia cases. Structural lung disease and hematologic malignancy were common underlying diseases. Overall death rate was $54.5 \%$. Higher death rates were associated with co-infection (83.3\%) and immunocompromise (80.0\%).

Tuman bocavirus (HBoV), a DNA virus in the 1 Parvoviridae family, was first identified in 2005 (1). $\mathrm{HBoV}$ is distributed worldwide and has been found in $2 \%-33 \%$ of respiratory specimens, primarily from children with acute respiratory tract infection $(2,3)$. In adults, $\mathrm{HBoV}$ is an uncommon cause of upper respiratory tract infection and pneumonia (4-6). It can also be associated with the acute exacerbation of chronic obstructive pulmonary disease (7). Recently, a few case reports have shown that $\mathrm{HBoV}$ can be associated with life-threatening pneumonia (8-12). However, severe $\mathrm{HBoV}$-associated pneumonia has not been reported in a case series, and little is known about the characteristics of $\mathrm{HBoV}$-associated pneumonia in critically ill adult patients. We investigated the incidence, clinical characteristics, and outcomes of severe $\mathrm{HBoV}$-associated pneumonia in adults in Seoul, South Korea.

\section{The Study}

We conducted a prospective observational cohort study of severe pneumonia in adult patients admitted to the medical intensive-care unit (ICU) at a 2,700-bed referral hospital in Seoul, South Korea, during March

Author affiliation: Asan Medical Center, University of Ulsan College of Medicine, Seoul, South Korea

DOI: https://doi.org/10.3201/eid2701.202061
2010-February 2019 (13,14). We initially included all adult patients admitted to the ICU with a diagnosis of pneumonia but later excluded patients with nonsevere pneumonia. We collected data on demographics, underlying diseases or conditions, immune status, seasonality, clinical manifestations, laboratory findings, pathogens, complications, treatment, and outcomes. This data collection was a routine part of the management and care of these patients at our hospital. Definitions and microbial evaluations are summarized in the Appendix (https://wwwnc.cdc. gov/EID/article/27/1/20-2061-App1.pdf). The Institutional Review Board of Asan Medical Center approved this study (approval no. 2010-0079) and waived informed-consent requirements.

During the study, 2,519 adult patients were admitted to the ICU with the diagnosis of severe pneumonia. After excluding 298 patients (83 community-acquired pneumonia [CAP] cases and 215 hospital-acquired pneumonia [HAP] cases) for whom multiplex respiratory virus PCR was not performed, 2,221 severe pneumonia patients $(1,482$ CAP cases and 739 HAP cases) were included. Among these 2,221 severe pneumonia patients, septic shock occurred in 1,306 (58.8\%), and 2,141 $(96.4 \%)$ required mechanical ventilation. Septic shock occurred in 80 patients $(3.6 \%)$ who did not require mechanical ventilation.

Mean patient age was 65.8 years (range 16-97 years). Structural lung disease $(26.9 \%)$ was the most common underlying disease in patients with CAP, and hematologic malignancy $(25.4 \%)$ was the most common in patients with HAP (Appendix Table 1). One or more respiratory pathogens were identified in 1,510 patients $(68.0 \%)$ (Appendix Table 2). Overall,

${ }^{1}$ These authors contributed equally to this article. 
$888(40.0 \%)$ patients had bacterial infections, 711 $(32.0 \%)$ had viral infections, and $230(10.4 \%)$ patients had bacterial-viral co-infection. A total of 787 viruses were identified in 711 patients. Two viruses were identified in 60 patients and 3 viruses in 8 patients. Influenza virus $(8.6 \%)$ and rhinovirus $(8.4 \%)$ were the most common viral pathogens for severe CAP, whereas parainfluenza virus and respiratory syncytial virus were the most common viral pathogens for severe HAP.

Eleven $\mathrm{HBoV}$-associated severe pneumonia cases $(0.5 \%[11 / 2,221])$ were reported. Of those, $\mathrm{HBoV}$ accounted for $0.4 \%(6 / 1,482)$ of CAP cases and $0.7 \%$ (5/739) of HAP cases. Of the 711 virus-associated severe pneumonia cases, $\mathrm{HBoV}$ accounted for $1.2 \%$ $(6 / 501)$ of CAP cases and $2.4 \%(5 / 210)$ of HAP cases. Appendix Table 3 summarizes the characteristics and outcomes of the 11 patients with $\mathrm{HBoV}$-associated severe pneumonia, which included 6 patients with CAP and 5 patients with HAP. HBoV occurred in all 4 seasons but was more common during September-February (8 cases). Nine patients were men $(81.8 \%)$; the median age was 69.0 years (range $36-81$ years). All patients had $\geq 1$ severe underlying diseases. Structural lung disease (5 patients) and hematologic malignancy (4 patients) were the most common underlying illnesses. Five patients $(45.5 \%)$ were immunocompromised.

Viruses were detected by using nasopharyngeal aspirate or swab specimens. In 1 patient, the virus was detected in bronchoalveolar lavage fluid and nasopharyngeal samples. Co-infection was observed in 6 patients $(54.5 \%)$. Eight patients underwent chest computed tomography. The most common radiologic findings were bilateral and multifocal consolidation and ground-glass opacity.

The median length of ICU stay was 9.0 days (range 1-74 days). Overall death rate was 54.5\% (6/11). The death rate was $80.0 \%(4 / 5)$ for immunocompromised patients and $33.3 \%(2 / 6)$ for immunocompetent patients. Higher death rates were observed in cases of co-infection $(83.3 \%, 5 / 6)$ than in cases of sole $\mathrm{HBoV}$ infection $(20.0 \%[1 / 5])(p=0.08)$. All immunocompromised patients with co-infection died (3/3), whereas no immunocompetent patients without co-infection died $(0 / 3)$.

\section{Conclusions}

Our study demonstrated that $\mathrm{HBoV}$ is an uncommon pathogen for adult patients with severe pneumonia requiring ICU admission. All episodes we investigated occurred in patients with serious underlying diseases, and co-infection was frequent. Overall death rates were high and closely associated with immunocompromised state and presence of co-infection.

Information on severe $\mathrm{HBoV}$-associated pneumonia in adults is limited. Five cases of severe HBoVassociated pneumonia have been reported to date (8-12), which included 3 cases from the same facility in Germany $(9,10,12)$. Of the 5 patients, 3 had hematologic malignancy and 1 had cystic fibrosis. One of the patients was a 74-year-old immunocompetent man (10). He had an acute head injury and rib fracture, probably because of weakness from $\mathrm{HBoV}$ pneumonia, necessitating mechanical ventilation. In our study, patients with structural lung diseases, including chronic obstructive pulmonary disease and bronchiectasis, were predisposed to severe $\mathrm{HBoV}$ associated pneumonia. Most of these patients were not immunocompromised and had CAP. Therefore, clinicians should consider $\mathrm{HBoV}$ as an uncommon pathogen of severe pneumonia in adults with structural lung disease.

Consistent with previous reports $(4,5)$, we found a high rate of co-infection with other pathogens in patients with $\mathrm{HBoV}$ infection. $\mathrm{HBoV}$ has shown a prolonged persistence in the mucosa of the respiratory tract. Viral persistence contributes to the high frequency of coinfections with proper respiratory pathogens (15). This phenomenon might be associated with the underlying severe diseases. Of note, co-infection was closely related to higher overall death rates in our patients. Our series included 5 cases of sole $\mathrm{HBoV}$ infection, which was more common in CAP patients and associated with lower death rates. These findings indicated that $\mathrm{HBoV}$ itself has a lower virulence potential and rarely causes severe pneumonia, which is predominant in immunocompromised patients or patients with underlying structural lung disease. The higher incidence of severe $\mathrm{HBoV}$-associated pneumonia in HAP patients compared with CAP patients $(0.7 \%$ vs. $0.4 \%)$ might be explained by the higher proportion of immunocompromised patients in the HAP population.

Our study has some limitations. First, we excluded 298 patients ( 83 of 1,565 CAP patients [5.8\%] and 215 of 954 HAP patients [22.5\%]) for whom multiplex respiratory virus PCR was not performed. Because our study was observational, microbial evaluations and patient-management decisions were made by attending physicians, and multiplex respiratory virus PCR test was not used for all patients. Therefore, selection bias might have occurred, especially for HAP. Second, HBoV was mostly identified through nasopharyngeal specimens only and was frequently accompanied by copathogens. Therefore, we could 
not evaluate in detail the virulence potential of sole $\mathrm{HBoV}$ infection. Finally, we did not conduct a genotypic study of $\mathrm{HBoV}$, and the viral load therefore was not tested.

In summary, in this study, $\mathrm{HBoV}$ accounted for $0.5 \%$ of severe pneumonia cases in adults. HBoV-associated severe pneumonia could lead to high death rates. Underlying severe diseases and frequent coinfection seem to be responsible for poor outcomes.

This work was supported by Asan Institute of Life Sciences (grant 2010-0079) and generous gifts from Sam Won Song.

\section{About the Author}

Dr. Choi is an infectious disease specialist and professor at Asan Medical Center, Ulsan University College of Medicine, in Seoul, South Korea. His main research interest is the epidemiology of severe pneumonia caused by respiratory viruses. Dr. Huh is an intensivist and professor at Asan Medical Center. Her main research interest is the pathogenesis and management of acute respiratory distress syndrome.

\section{References}

1. Allander T, Tammi MT, Eriksson M, Bjerkner A, Tiveljung-Lindell A, Andersson B. Cloning of a human parvovirus by molecular screening of respiratory tract samples. Proc Natl Acad Sci U S A. 2005;102:12891-6. https:/ / doi.org/10.1073/pnas.0504666102

2. Jartti T, Hedman K, Jartti L, Ruuskanen O, Allander T, Söderlund-Venermo M. Human bocavirus - the first 5 years. Rev Med Virol. 2012;22:46-64. https://doi.org/10.1002/ rmv.720

3. Choi EH, Lee HJ, Kim SJ, Eun BW, Kim NH, Lee JA, et al. The association of newly identified respiratory viruses with lower respiratory tract infections in Korean children, 2000-2005. Clin Infect Dis. 2006;43:585-92. https:/ / doi.org/ $10.1086 / 506350$

4. Liu WK, Chen DH, Liu Q, Liang HX, Yang ZF, Qin S, et al. Detection of human bocavirus from children and adults with acute respiratory tract illness in Guangzhou, southern China. BMC Infect Dis. 2011;11:345. https:/ / doi.org/10.1186/ 1471-2334-11-345

5. Lee HN, Koo HJ, Kim SH, Choi SH, Sung H, Do KH. Human bocavirus infection in adults: clinical features and radiological findings. Korean J Radiol. 2019;20:1226-35. https:/ / doi.org/10.3348/kjr.2018.0634

6. Gu K, Van Caeseele P, Dust K, Ho J. Atypical pneumonia due to human bocavirus in an immunocompromised patient. CMAJ. 2017;189:E697-9. https://doi.org/10.1503/ cmaj.161134

7. Longtin J, Bastien M, Gilca R, Leblanc E, de Serres G, Bergeron MG, et al. Human bocavirus infections in hospitalized children and adults. Emerg Infect Dis. 2008;14:217-21. https://doi.org/10.3201/eid1402.070851

8. Kupfer B, Vehreschild J, Cornely O, Kaiser R, Plum G, Viazov $S$, et al. Severe pneumonia and human bocavirus in adult. Emerg Infect Dis. 2006;12:1614-6. https://doi.org/ 10.3201/eid1210.060520

9. Krakau M, Brockmann M, Titius B, Limmroth C, Khalfaoui S, Schildgen $\mathrm{V}$, et al. Acute human bocavirus infection in MDS patient, Cologne, Germany. J Clin Virol. 2015;69:44-7. https:// doi.org/10.1016/j.jcv.2015.05.029

10. Krakau M, Gerbershagen K, Frost U, Hinzke M, Brockmann M, Schildgen V, et al. Case report: human bocavirus associated pneumonia as cause of acute injury, Cologne, Germany. Medicine (Baltimore). 2015;94:e1587. https:/ / doi.org/10.1097/MD.0000000000001587

11. Sadeghi M, Kantola K, Finnegan DP, McCaughey C, Hedman L, Söderlund-Venermo M, et al. Possible involvement of human bocavirus 1 in the death of a middle-aged immunosuppressed patient. J Clin Microbiol. 2013;51:3461-3. https://doi.org/10.1128/JCM.01157-13

12. Dieninghoff D, Karagiannidis C, Straßmann S, Pieper M, Dammaschek S, Zabner J, et al. Fatal HBoV-1 infection in adult female cystic fibrosis patient. Hum Pathol (N Y). 2017;7:51-2. https:// doi.org/10.1016/j.ehpc.2016.07.001

13. Choi SH, Hong SB, Ko GB, Lee Y, Park HJ, Park SY, et al. Viral infection in patients with severe pneumonia requiring intensive care unit admission. Am J Respir Crit Care Med. 2012;186:325-32. https:/ / doi.org/10.1164/ rccm.201112-2240OC

14. Hong HL, Hong SB, Ko GB, Huh JW, Sung H, Do KH, et al. Viral infection is not uncommon in adult patients with severe hospital-acquired pneumonia. PLoS One. 2014;9:e95865. https://doi.org/10.1371/journal.pone.0095865

15. Broccolo F, Falcone V, Esposito S, Toniolo A. Human bocaviruses: possible etiologic role in respiratory infection. J Clin Virol. 2015;72:75-81. https:/ / doi.org/10.1016/ j.jcv.2015.09.008

Address for correspondence: Younsuck Koh, Department of Pulmonary and Critical Care Medicine, Asan Medical Center, University of Ulsan College of Medicine, 88, Olympic-ro 43-gil, Songpa-gu, Seoul, 138-736, South Korea; email: yskoh@amc.seoul.kr 